\title{
ORIGINAL
}

\section{Relationship between SARS-CoV-2 infection and the incidence of ventilator-associated lower respiratory tract infections: a European multicenter cohort study}

Anahita Rouzé ${ }^{1,2}$, Ignacio Martin-Loeches ${ }^{3,4}$, Pedro Povoa ${ }^{5,6}$, Demosthenes Makris ${ }^{7}$, Antonio Artigas ${ }^{8}$, Mathilde Bouchereau ${ }^{1}$, Fabien Lambiotte ${ }^{9}$, Matthieu Metzelard ${ }^{10}$, Pierre Cuchet $^{11}$, Claire Boulle Geronimi ${ }^{12}$, Marie Labruyere ${ }^{13}$, Fabienne Tamion ${ }^{14}$, Martine Nyunga ${ }^{15}$, Charles-Edouard Luyt ${ }^{16}$, Julien Labreuche ${ }^{17}$, Olivier Pouly ${ }^{18}$, Justine Bardin ${ }^{19}$, Anastasia Saade ${ }^{20}$, Pierre Asfar ${ }^{21}$, Jean-Luc Baudel ${ }^{22}$, Alexandra Beurton ${ }^{23}$, Denis Garot ${ }^{24}$, Iliana loannidou ${ }^{25}$, Louis Kreitmann ${ }^{26}$, Jean-François Llitjos ${ }^{27}$, Eleni Magira ${ }^{28}$, Bruno Mégarbane ${ }^{29}$, David Meguerditchian ${ }^{30}$, Edgar Moglia ${ }^{31}$, Armand Mekontso-Dessap ${ }^{32}$, Jean Reignier ${ }^{33}$, Matthieu Turpin ${ }^{34}$, Alexandre Pierre ${ }^{35}$, Gaetan Plantefeve ${ }^{36}$, Christophe Vinsonneau ${ }^{37}$, Pierre-Edouard Floch ${ }^{38}$, Nicolas Weiss ${ }^{39}$, Adrian Ceccato ${ }^{40}$, Antoni Torres ${ }^{41}$, Alain Duhamel ${ }^{17}$, Saad Nseir ${ }^{1,2^{*}}$ (D) on behalf of the coVAPid study Group

(c) 2021 Springer-Verlag GmbH Germany, part of Springer Nature

\begin{abstract}
Purpose: Although patients with SARS-CoV-2 infection have several risk factors for ventilator-associated lower respiratory tract infections (VA-LRTI), the reported incidence of hospital-acquired infections is low. We aimed to determine the relationship between SARS-CoV-2 pneumonia, as compared to influenza pneumonia or no viral infection, and the incidence of VA-LRTI.

Methods: Multicenter retrospective European cohort performed in $36 \mathrm{ICUs}$. All adult patients receiving invasive mechanical ventilation $>48 \mathrm{~h}$ were eligible if they had: SARS-CoV-2 pneumonia, influenza pneumonia, or no viral infection at ICU admission. VA-LRTI, including ventilator-associated tracheobronchitis (VAT) and ventilator-associated pneumonia (VAP), were diagnosed using clinical, radiological and quantitative microbiological criteria. All VA-LRTI were prospectively identified, and chest- $X$ rays were analyzed by at least two physicians. Cumulative incidence of first episodes of VA-LRTI was estimated using the Kalbfleisch and Prentice method, and compared using Fine-and Gray models.

Results: 1576 patients were included (568 in SARS-CoV-2, 482 in influenza, and 526 in no viral infection groups). VALRTI incidence was significantly higher in SARS-CoV-2 patients (287, 50.5\%), as compared to influenza patients (146, $30.3 \%$, adjusted sub hazard ratio (sHR) 1.60 (95\% confidence interval (CI) 1.26 to 2.04)) or patients with no viral infection (133, 25.3\%, adjusted sHR 1.7 (95\% Cl 1.2 to 2.39)). Gram-negative bacilli were responsible for a large proportion (82\% to $89.7 \%$ ) of VA-LRTI, mainly Pseudomonas aeruginosa, Enterobacter spp., and Klebsiella spp.
\end{abstract}

\footnotetext{
*Correspondence: s-nseir@chru-lille.fr

${ }^{1} \mathrm{CHU}$ de Lille, Médecine Intensive-Réanimation, 59000 Lille, France

Full author information is available at the end of the article
}

\section{国 Springer}


Conclusions: The incidence of VA-LRTI is significantly higher in patients with SARS-CoV-2 infection, as compared to patients with influenza pneumonia, or no viral infection after statistical adjustment, but residual confounding may still play a role in the effect estimates.

Keywords: SARS-CoV-2, COVID-19, Ventilator-associated pneumonia, Ventilator-associated tracheobronchitis, Critical illness

\section{Introduction}

The novel severe acute respiratory syndrome coronavirus 2 (SARS-CoV-2) is the cause of a rapidly spreading illness, Coronavirus Disease 2019 (COVID-19), affecting millions of people worldwide. A large percentage (17$32 \%$ ) of patients hospitalized for SARS-CoV-2 infection are admitted to the intensive care unit (ICU) [1-3]. In spite of frequent use of high flow nasal oxygen and noninvasive mechanical ventilation $(55 \%$, and $16 \%$, respectively), intubation and invasive mechanical ventilation are required in approximately $10 \%$ of patients hospitalized for SARS-CoV-2-related infection [3].

Ventilator-associated lower respiratory tract infections (VA-LRTI), including ventilator-associated tracheobronchitis (VAT), and ventilator-associated pneumonia (VAP) are common in critically ill patients receiving invasive mechanical ventilation [4]. These infections are associated with increased duration of mechanical ventilation and ICU stay [5]. In addition, VAP is associated with increased mortality and cost [6]. Patients with SARS$\mathrm{CoV}-2$ pneumonia could be at increased risk for VALRTI, because of acute respiratory distress syndrome (ARDS), and the long duration of mechanical ventilation. ARDS is a well-known risk factor for VAP [7, 8], and its incidence in mechanically ventilated patients with SARS$\mathrm{CoV}-2$ pneumonia was reported to be as high as $42-89 \%$ $[1,9]$. In addition, immunosuppressive agents are commonly used in patients with SARS-CoV-2 infection [1], and SARS-CoV-2 is responsible for altered immune response [10-12]. However, based on the scarce available data, the incidence of hospital-acquired infections (HAI) appears to be low (13.5\%) [13]. The low incidence of HAI could be explained by strict hygiene and isolation measures, alteration of gut microbiota induced by SARS$\mathrm{CoV}-2$, and less pronounced innate immune suppression in these patients [14-16]. To our knowledge, no study to date has specifically addressed the impact of SARS$\mathrm{CoV}-2$ infection on the incidence of VA-LRTI.

We hypothesized that the incidence of VA-LRTI would be lower in patients with SARS-CoV-2 pneumonia, as compared to those with influenza pneumonia, or no viral infection. Therefore, we conducted this multicenter cohort study to determine the relationship between SARS-CoV-2 infection and the incidence, and etiology of VA-LRTI.

\section{Take-home message}

The incidence of VA-LRTI is significantly higher in patients with SARS-CoV-2 infection, as compared to patients with influenza pneumonia, or no viral infection.

\section{Methods \\ Study design and population}

This multicenter retrospective observational cohort study was conducted in 36 ICUs in Europe (28 centers in France, 3 in Spain, 3 in Greece, 1 in Portugal and 1 in Ireland), selected by invitation. Eligibility criteria included age equal or above 18 years, the need for invasive mechanical ventilation for more than $48 \mathrm{~h}$, and one of the following criteria at ICU admission: (1) SARS-CoV-2 pneumonia, (2) influenza (A or B) pneumonia, or (3) no viral infection. Exclusion criteria included age $<18$ years, duration of mechanical ventilation equal or less than $48 \mathrm{~h}$, and the presence of viral infection, other than SARS-CoV-2 or influenza, at ICU admission.

Participating centers retrospectively collected data from consecutive patients admitted to their ICU with SARS-CoV-2, influenza pneumonia, or no viral infection. Centers were invited to include the same number of patients in each of the three study groups (up to 20 patients per group, and 60 patients in total).

In the SARS-CoV-2 group, consecutive patients with SARS-CoV-2 pneumonia were included, starting at the beginning of the COVID-19 pandemic in each center. SARS-CoV-2 infection was confirmed by positive polymerase chain reaction (PCR) testing of a nasopharyngeal or respiratory secretions samples. In the influenza group, consecutive patients with influenza A or B pneumonia were included starting from the last patients admitted with this diagnosis this year and going back to previous seasons if necessary. Influenza pneumonia was diagnosed based on a positive nasopharyngeal or airway secretions PCR test. In the third group, consecutive patients admitted to the ICU for any reason, with no viral infection at ICU admission, were included from January 31, 2020 back to 2019 if necessary.

The Ethics Committee, and Institutional Review Boards approved the study protocol (Comite de Protection des Personnes Ouest VI; approved by April 14, 
2020; registration number RIPH:20.04.09.60039) as minimal-risk research using data collected for routine clinical practice, and waived the requirement for informed consent. In accordance with the French law, the database was registered into the "Commission Nationale l'Informatique et des Libertés". Based on CNIL request, patients or their proxies received information about the study and were given the possibility to refuse using their personal data. This was not required in countries other than France. The study was registered at ClinicalTrials. gov, number NCT04359693.

\section{Data collection}

Patient demographic characteristics, severity scores, comorbidities, reasons for ICU admission, prior hospitalization or antibiotic exposure (during the three months preceding ICU admission) were recorded at baseline for all patients. Data on antibiotic, antiviral and steroid treatments received during ICU stay, as well as prone positioning or use of extracorporeal membrane oxygenation (ECMO), were obtained. Further, clinical, biological, radiological and microbiological diagnostic criteria for VA-LRTI, and clinical outcomes (duration of mechanical ventilation, ICU length of stay, ICU mortality and 28-day mortality) were collected. Patients were followed up until day 28 or ICU discharge, if it occurred before.

\section{Definitions \\ Ventilator-associated lower respiratory tract infection}

The diagnosis of VA-LRTI was based on the presence of at least two of the following criteria: body temperature of more than $38.5{ }^{\circ} \mathrm{C}$ or less than $36.5{ }^{\circ} \mathrm{C}$, leucocyte count greater than 12000 cells per $\mu \mathrm{L}$ or less than 4000 cells per $\mu \mathrm{L}$, and purulent tracheal secretions [4]. Additionally, all episodes of infection needed microbiological confirmation, with the isolation in the endotracheal aspirate of at least $10^{5}$ colony-forming units (CFU) per $\mathrm{mL}$, or in bronchoalveolar lavage of at least $10^{4} \mathrm{CFU}$ per mL. VAT was defined with the above-mentioned criteria with no radiographic signs of new pneumonia. VAP was defined by the presence of new or progressive infiltrates on chest X-ray. Only first episodes of VAT and VAP occurring more than $48 \mathrm{~h}$ after starting invasive mechanical ventilation were analyzed. VAP was defined as occurring subsequently to VAT if it was diagnosed in the $96 \mathrm{~h}$ period after diagnosis of VAT and if the same microorganism caused both infections. All VA-LRTI episodes were prospectively identified, and chest X-rays, and other definition criteria were reviewed by at least two attending physicians. In case of disagreement, a third physician was asked to interpret the radiograph.

\section{Antibiotic treatment and microbiological data}

Antibiotic treatment was considered appropriate when at least one antibiotic, matching the in vitro susceptibility of the pathogen causing VA-LRTI, was administered to treat this infection [17]. Microbiological identification and susceptibility tests were performed using standard methods. Multidrug resistant (MDR) isolates were defined as acquired non-susceptibility to at least one agent in three or more antimicrobial categories [18].

\section{Outcomes}

The primary outcome of our study was the incidence of VA-LRTI, including VAT and VAP, among patients admitted to ICU with SARS-CoV-2 infection, as compared to Influenza and no viral infection groups. The secondary endpoint was the etiology of VA-LRTI.

\section{Statistical analysis}

We planned to include a total of 1071 patients (357 per group) to have a statistical power of $80 \%$ to demonstrate, with a two-sided test at 0.025 level (to consider the two comparisons by applying a Bonferroni correction), a difference of $10 \%$ in 28-day cumulative incidence of first episodes of VA-LRTI in favor of SARS-CoV-2 pneumonia group against Influenza pneumonia or no viral infection groups. The sample size was calculated using PASS 12 (Logrank Test Accounting for Competing Risks) [19] on the basis of an expected rate of $20 \%$ in the two nonSARS-CoV-2 groups and $10 \%$ in SARS-CoV-2 group and an expected competing event rate of $30 \%$ in non-SARS$\mathrm{CoV}-2$ groups and $50 \%$ in SARS-CoV-2 group [4].

Quantitative variables were expressed as medians (interquartile range) and categorical variables were expressed as numbers (percentage). Patient characteristics at ICU admission and during ICU stay were described according to study groups (SARS-CoV-2 pneumonia vs. Influenza pneumonia vs. no viral infection) without formal statistical comparisons.

The 28-day cumulative incidence of first episodes of VA-LRTI (VA-LRTI, VAT, VAP) were estimated using the Kalbfleisch and Prentice method [20], considering extubation within 28-day (dead or alive) as a competing event. For VAT and VAP incidence, occurrence of VAP and VAT was respectively treated as a competing event, in addition to extubation. Comparison of the incidence of first episodes of VA-LRTI between study groups was done using Gray' test to consider competing events. Subhazard ratios (and their 95\% confidence intervals (CIs)) associated with SARS-CoV-2 pneumonia, against each other group, were calculated using univariable Fine-and Gray models as effect sizes. Comparisons were further adjusted for pre-specified confounders known to be associated with VA-LRTI incidence (age, gender, SAPS 
II, MacCabe classification, Immunosuppression, recent hospitalization, recent antibiotic, and ARDS) [8] by using multivariable Fine-and Gray models. Regarding the causal relationship, we also assessed the association of VA-LRTI with SARS-CoV-2 pneumonia using univariable and multivariable cause-specific Cox's proportional hazard models.

To avoid case-deletion in multivariate analyses due to presence of missing data in covariates, multivariable Fine-and Gray models, and cause-specific Cox's, were performed after handling missing data on patient's characteristics at ICU admission using multiple imputation procedure [21]. Imputation procedure was performed using regression switching approach (chained equations with $m=20$ imputations obtained) under the missing at random assumption by considering outcomes (event status and log of event time) and all baseline characteristics (see ESM Table 1 for missing data pattern and see ESM Table 2 for baseline data in complete and incomplete cases) in imputed models. Predictive mean matching method, and logistic regression model (binary, ordinal or multinomial) were used for quantitative, and categorical variables; respectively. We used 20 imputations, allowing a maximal fraction of missing information (FMI) $/ \mathrm{m}<0.1$ in all analyses (see ESM Table 3). Estimates obtained in the different imputed data sets were combined using Rubin's rules [22].

Data were analyzed using the SAS software package, release 9.4 (SAS Institute, Cary, NC).

\section{Results}

In total, 1576 patients were included (from March 2016 through May 2020) in the 36 participating centers (568 in SARS-CoV-2 pneumonia group, 482 in influenza pneumonia group, and 526 in no viral infection group).

\section{Patient characteristics at ICU admission}

The percentage of medical admission category, ARDS, and body mass index (BMI) were higher in SARS-CoV-2 pneumonia group, as compared to the two other groups. SAPS II, SOFA score, comorbidities, and ratio of recent hospitalization, recent antimicrobial treatment, shock, cardiac arrest, neurological or acute kidney injury were lower in SARS-CoV-2 pneumonia group, as compared to the two other groups (Table 1, and ESM Fig. 1). Influenza $A$ and $B$ were diagnosed in 421 (87.3\%), and 61 (12.7\%) of patients with influenza pneumonia, respectively.

\section{Patient characteristics during ICU stay}

Corticosteroids, exposure to antimicrobials, and ECMO were comparable in SARS-CoV-2 and influenza pneumonia groups, but higher compared to the no viral infection group. Duration of exposure to antimicrobials was comparable in the three groups. The dose and duration of corticosteroids were higher in SARS-CoV-2 pneumonia group, as compared to the other groups. Prone positioning was more common in SARS-CoV-2 pneumonia group, compared to other groups. Duration of mechanical ventilation and ICU stay were longer in SARS-CoV-2 pneumonia group, compared to other groups. ICU mortality and 28-day mortality were comparable in the three groups (Table 2). Total duration of antimicrobial treatment was longer in SARS-CoV-2 patients, as compared to patients with influenza, or no viral infection (Median (IR) 12 days $(7,18), 10(7,18), 8(5,15)$; respectively).

\section{Incidence of VA-LRTI}

A large proportion of VA-LRTI was diagnosed after the first week of mechanical ventilation.

The incidence of VA-LRTI was significantly higher in SARS-CoV-2 pneumonia group than in the two other groups (Table 3, Fig. 1, $p$ value adjusted for multiple comparison using Bonferroni method $p \leq 0.001$ ). This difference remained significant after adjustment for pre-specified confounding factors at ICU admission in both multiple imputation analyses using Fine-and Gray models, the one after (subhazard ratio $(95 \% \mathrm{CI})$ 1.60 (1.26-2.04) for SARS-CoV-2 vs. Influenza and 1.70 (1.20-2.39) for SARS-CoV-2 vs. No viral infection), as well as the other using complete-available cases (ESM Table 4). However, the association was reduced in cause-specific analysis with an adjusted cHRs (95\% CI) of 1.41 (1.10-1.80) for SARS-CoV-2 vs. Influenza and 1.24 (0.97-1.59) for SARS-CoV-2 vs. no viral infection. The cumulative incidence of VAT, and VAP (as first events) was also higher in SARS-CoV-2 pneumonia group than in the two other groups (Table 3 ). However, in multivariate analysis, only cumulative incidence of VAP remained significantly higher in SARS-CoV-2 group compared to two other groups (Table 3), this association was also reduced in cause-specific specific analysis (ESM Table 5).

\section{Characteristics of patients with VA-LRTI}

In the three study groups, SOFA score, clinical pulmonary infection score (CPIS), $\mathrm{PaO}_{2} / \mathrm{FiO}_{2}$, the rate of bronchoalveolar lavage, antibiotic treatment, and appropriate antibiotic treatment were lower in patients with VAT, as compared to those with VAP (Table 4). Transition from VAT to VAP was diagnosed in 21 (12.6\%) of 167 patients (including 10 of 82 (12.2\%), 2 of $39(5.1 \%)$, and 9 of $46(19.6 \%)$ in patients with SARSCoV-2 pneumonia, influenza pneumonia, and no viral infection; respectively). 
Table 1 Patient characteristics at ICU admission

\begin{tabular}{|c|c|c|c|}
\hline & $\begin{array}{l}\text { SARS-CoV-2 pneumonia } \\
(n=568)\end{array}$ & $\begin{array}{l}\text { Influenza pneumonia } \\
(n=482)\end{array}$ & $\begin{array}{l}\text { No viral infection } \\
(n=526)\end{array}$ \\
\hline Age, years ${ }^{a}$ & $64(55-71)$ & $62(53-71)$ & $65(55-74)$ \\
\hline Men & $407 / 568(71.7)$ & 298/482 (61.8) & $353 / 524(67.4)$ \\
\hline Body mass index, $\mathrm{kg} / \mathrm{m}^{2 \mathrm{~b}}$ & $28.9(25.8-33.3)$ & $27.5(23.3-32.3)$ & $26.5(22.8-31)$ \\
\hline \multicolumn{4}{|l|}{ Severity scores } \\
\hline SAPS $\|^{C}$ & $41(32-55)$ & $50(39-64)$ & $56(42-67)$ \\
\hline SOFA score ${ }^{d}$ & $6(3-8)$ & $8(6-11)$ & $9(6-11)$ \\
\hline \multicolumn{4}{|l|}{ Comorbidity scores } \\
\hline \multicolumn{4}{|l|}{ MacCabe classification } \\
\hline Non-fatal & $475 / 543(87.5)$ & $324 / 456(71.1)$ & $315 / 489(64.4)$ \\
\hline Fatal $<5$ years & $62 / 543(11.4)$ & $114 / 456(25)$ & $137 / 489(28)$ \\
\hline Fatal $<1$ year & $6 / 543(1.1)$ & 18/456 (3.9) & $37 / 489(7.6)$ \\
\hline Charlson Comorbidity Index & $3(1-4)$ & $3(2-5)$ & $4(2-6)$ \\
\hline \multicolumn{4}{|l|}{ Chronic diseases } \\
\hline Diabetes mellitus & $168 / 565(29.7)$ & 104/474 (21.9) & $132 / 519(25.4)$ \\
\hline Chronic renal failure & $33 / 559(5.9)$ & $39 / 475(8.2)$ & $45 / 521(8.6)$ \\
\hline Heart disease & $103 / 560(18.4)$ & $117 / 476(24.6)$ & $134 / 518(25.9)$ \\
\hline Chronic heart failure & 21/558 (3.8) & $37 / 475(7.8)$ & $50 / 518(9.7)$ \\
\hline COPD & $37 / 560(6.6)$ & $129 / 475(27.2)$ & $98 / 521(18.8)$ \\
\hline Chronic respiratory failure & 20/558 (3.6) & $67 / 475(14.1)$ & $49 / 518(9.5)$ \\
\hline Cirrhosis & $8 / 559(1.4)$ & $16 / 475(3.4)$ & $36 / 516(7)$ \\
\hline Immunosuppression & $52 / 559(9.3)$ & $107 / 479(22.3)$ & $117 / 520(22.5)$ \\
\hline Active smoking & $29 / 560(5.2)$ & $149 / 476(31.3)$ & $137 / 519(26.4)$ \\
\hline Alcohol abuse & $34 / 558(6.1)$ & 85/475 (17.9) & $132 / 519(25.4)$ \\
\hline \multicolumn{4}{|l|}{ Location before ICU admission } \\
\hline Home & $271 / 568(47.7)$ & 275/481 (57.2) & $265 / 526(50.4)$ \\
\hline Hospital ward & 215/568 (37.9) & 157/481 (32.6) & $230 / 526(43.7)$ \\
\hline Another ICU & $82 / 568(14.4)$ & $49 / 481(10.2)$ & $31 / 526(5.9)$ \\
\hline \multicolumn{4}{|l|}{ Admission category } \\
\hline Medical & $567 / 568(99.8)$ & $480 / 482(99.6)$ & $467 / 526(88.8)$ \\
\hline Surgical & $0 / 568(0)$ & $0 / 482(0)$ & $17 / 526(3.2)$ \\
\hline Trauma & 1/568 (0.2) & 2/482 (0.4) & $42 / 526(8)$ \\
\hline Recent hospitalization (<3 months) & $44 / 566(7.8)$ & $72 / 479(15)$ & $148 / 524(28.2)$ \\
\hline Recent antibiotics (<3 months) & $74 / 567(13.1)$ & 95/477 (19.9) & 103/524 (19.7) \\
\hline \multicolumn{4}{|l|}{ Causes for ICU admission } \\
\hline Shock & 102/557 (18.3) & $210 / 470(44.7)$ & $244 / 515(47.4)$ \\
\hline Acute respiratory failure & $521 / 567(91.9)$ & $433 / 461(90.2)$ & $279 / 505(54.4)$ \\
\hline ARDS & $386 / 563$ (68.6) & 220/469 (46.9) & 72/509 (14.1) \\
\hline Neurological failure & $26 / 548(4.7)$ & $69 / 465$ (14.8) & $191 / 509$ (37.5) \\
\hline Cardiac arrest & 3/547 (0.6) & $25 / 465(5.4)$ & $84 / 508(16.5)$ \\
\hline Acute renal failure & $96 / 567(17.5)$ & $133 / 480(28.9)$ & $136 / 513(26.9)$ \\
\hline
\end{tabular}

Values are as no./No.(\%) or median (interquartile range)

McCabe classification of comorbidities and likelihood of survival, likely to survive $>5$ years, $1-5$ years, $<1$ year; Chronic renal failure, KDOQI CKD classification stage 4 or 5 (creatinine clearance $<30 \mathrm{ml} / \mathrm{mn}$ ); Chronic heart failure, NYHA class III or IV; Heart disease, ischemic heart disease or atrial fibrillation; Cirrhosis, Child-Pugh score B or $\mathrm{C}$; Immunosuppression if cancer, hematological malignancy, allogeneic stem cell transplant, organ transplant, HIV or immunosuppressive drugs; More than one cause for ICU admission is possible

ARDS Acute respiratory Distress Syndrome, COPD Chronic Obstructive Pulmonary Disease, ICU Intensive Care Unit, SAPS II Simplified Acute Physiology Score II, SOFA Sequential Organ Failure Assessment

a 1 missing value in influenza group

b 160 missing values (SARS-CoV-2, $n=32$; influenza, $n=68$; controls, $n=60$ )

c 87 missing values (SARS-CoV-2, $n=43$; influenza, $n=21$; controls, $n=21$ )

d 27 missing values (SARS-CoV-2, $n=21$; influenza, $n=4$; controls, $n=2$ )

e 50 missing values (SARS-CoV-2, $n=19$; influenza, $n=11$; controls, $n=20$ ) 
Table 2 Patient characteristics during ICU stay

\begin{tabular}{|c|c|c|c|}
\hline & $\begin{array}{l}\text { SARS-CoV-2 pneumonia } \\
(n=568)\end{array}$ & $\begin{array}{l}\text { Influenza pneumonia } \\
(n=482)\end{array}$ & $\begin{array}{l}\text { No viral infection } \\
(n=526)\end{array}$ \\
\hline Antiviral treatment & $322 / 566(56.9)$ & $437 / 481(90.9)$ & $24 / 523(4.6)$ \\
\hline Oseltamivir & $44 / 563(7.8)$ & $430 / 475(90.5)$ & $22 / 521(4.2)$ \\
\hline Remdesivir & $27 / 563(4.8)$ & $0 / 475(0)$ & $0 / 521(0)$ \\
\hline Lopinavir-Ritonavir & $147 / 563(26.1)$ & $0 / 475(0)$ & $0 / 521(0)$ \\
\hline Lopinavir-Ritonavir + interferon & $21 / 563(3.7)$ & $0 / 475(0)$ & $0 / 521(0)$ \\
\hline Hydroxychloroquine & $173 / 563(30.7)$ & $1 / 475(0.2)$ & $0 / 521(0)$ \\
\hline Corticosteroids & $202 / 542(37.3)$ & 182/475 (38.3) & $161 / 525(30.7)$ \\
\hline Hydrocortisone & $59 / 537(11)$ & $107 / 473(22.6)$ & $80 / 520(15.4)$ \\
\hline Dexamethasone & $48 / 537(8.9)$ & $1 / 473(0.2)$ & $10 / 520(1.9)$ \\
\hline Methylprednisolone & $91 / 537(16.9)$ & $73 / 473(15.4)$ & $68 / 520(13.1)$ \\
\hline Highest daily dose, mga & $100(50-133)$ & $50(50-100)$ & $50(50-100)$ \\
\hline Duration, days ${ }^{b}$ & $6(4-9)$ & $5(3-9)$ & $4(3-7)$ \\
\hline Antibiotic treatment & $506 / 531(95.3)$ & $434 / 458(94.8)$ & 415/496 (83.7) \\
\hline Duration, days ${ }^{c}$ & $7(5-9)$ & $7(4-10)$ & $7(4-9)$ \\
\hline Prone positioning & $383 / 567(67.5)$ & 151/481 (31.4) & $63 / 522(12.1)$ \\
\hline ECMO & $61 / 567(10.8)$ & $60 / 480(12.5)$ & $5 / 523(1)$ \\
\hline \multicolumn{4}{|l|}{ 28-day outcomes } \\
\hline MV duration, days & $15(9-23)$ & $10(6-21)$ & $8(5-16)$ \\
\hline Ventilator-free days & $4(0-16)$ & $10(0-21)$ & $12(0-22)$ \\
\hline ICU length of stay, days & $18(12-27)$ & $14.0(8-27)$ & $12(7-21)$ \\
\hline ICU-free days & $0(0-12)$ & $2(0-17)$ & $4(0-17)$ \\
\hline ICU mortality & $164 / 568(28.9)$ & $125 / 482(25.9)$ & $173 / 526(32.9)$ \\
\hline 28-day mortality & $166 / 568(29.2)$ & $132 / 482(27.4)$ & $182 / 526(34.6)$ \\
\hline
\end{tabular}

Vales are no./No. (\%) or median (interquartile range)

In patients with VA-LRTI, duration of exposure to antibiotic treatment and corticosteroids was only taken before VA-LRTI. Corticosteroid regimens are reported as prednisone equivalent

ECMO Extracorporeal Membrane Oxygenation, ICU Intensive Care Unit, MV mechanical ventilation

a 11 missing values (SARS-CoV-2, $n=4$; influenza, $n=4$; controls, $n=3$ )

b 16 missing values (SARS-CoV-2, $n=7$; influenza, $n=3$; controls, $n=6$ )

c duration of antibiotic treatment was taken into account before VA-LRTI

\section{Microbiological results}

Gram-negative bacilli were responsible for the majority of VA-LRTI first episodes in the three study groups. Pseudomonas aeruginosa, Enterobacter spp., and Klebsiella spp. were the most commonly identified bacteria (Table 5). The rate of patients with VA-LRTI related to MDR bacteria was lower in SARS-CoV-2 pneumonia group, as compared to the two other groups.

\section{Discussion}

The main results of our study are that the incidence of VA-LRTI is significantly higher in patients with SARSCoV-2 pneumonia, as compared to patients with influenza pneumonia, or no viral infection at ICU admission. Gram-negative bacilli, mainly $P$. aeruginosa, Enterobacter spp., and Klebsiella spp., were responsible for the majority of VA-LRTI first episodes. MDR bacteria were less common in SARS-CoV-2 patients with VA-LRTI, as compared to the two other groups.

Previous studies, not specifically designed to evaluate the relationship between SARS-CoV-2 infection and VA-LRTI, reported low incidence of hospital-acquired pneumonia (11.5\%) [13], and comparable incidence of VAP (25\%) [1]. However, no clear definition was given for hospital-acquired pneumonia or VAP, and whether quantitative microbiological confirmation was required is unknown. Further, a relatively small number of SARSCoV-2 patients were included in these studies, no control group was used, and no adjustment was performed for confounding factors.

Several potential explanations could be provided for the high incidence of VA-LRTI in patients with SARS-CoV-2 pneumonia. First, patients with SARSCoV-2 pneumonia had longer duration of mechanical 
Table 3 Incidence of first episodes of ventilator-associated lower respiratory tract infections

\begin{tabular}{|c|c|c|c|c|c|c|c|c|}
\hline & \multirow[b]{2}{*}{$\begin{array}{l}\text { SARS- } \\
\text { CoV-2 } \\
(n=568)\end{array}$} & \multirow[b]{2}{*}{$\begin{array}{l}\text { Influenza } \\
(n=482)\end{array}$} & \multirow[b]{2}{*}{$\begin{array}{l}\text { No viral } \\
\text { infection } \\
(n=526)\end{array}$} & \multirow[b]{2}{*}{$p$ value $^{a}$} & \multicolumn{2}{|c|}{ Unadjusted SHR (95\%Cl) } & \multicolumn{2}{|c|}{ Adjusted SHR $(95 \% \mathrm{Cl})^{e}$} \\
\hline & & & & & $\begin{array}{l}\text { SARS-CoV-2 vs. } \\
\text { Influenza }\end{array}$ & $\begin{array}{l}\text { SARS-CoV-2 vs. } \\
\text { No viral infec- } \\
\text { tion }\end{array}$ & $\begin{array}{l}\text { SARS-CoV-2 vs. } \\
\text { Influenza }\end{array}$ & $\begin{array}{l}\text { SARS-CoV-2 vs. } \\
\text { No viral infec- } \\
\text { tion }\end{array}$ \\
\hline VALRTI & $287(50.5)$ & $146(30.3)$ & $133(25.3)$ & $<0.0001$ & $1.87(1.53-2.27)^{\mathrm{b}}$ & $2.27(1.84-2.79)^{b}$ & $1.60(1.26-2.04)^{b}$ & $1.7(1.2-2.39)^{b}$ \\
\hline$V_{A} T^{c}$ & $82(14.4)$ & $39(8.1)$ & $46(8.8)$ & 0.0001 & $1.83(1.25-2.68)^{b}$ & $1.69(1.18-2.43)^{b}$ & $1.50(0.89-2.54)$ & $1.25(0.7-2.2)$ \\
\hline$V_{A P}^{d}$ & $205(36.1)$ & $107(22.2)$ & $87(16.5)$ & $<0.0001$ & $1.74(1.38-2.2)^{b}$ & $2.38(1.84-3.06)^{b}$ & $1.57(1.2-2.04)^{b}$ & $1.84(1.26-2.7)^{b}$ \\
\hline
\end{tabular}

Values are number of first events (28-day cumulative incidence expressed as \%, considering extubations (death or alive) as competing events) SHR calculated using marginal Fine and Gray's models for clustered (center) data

ARDS Acute respiratory Distress Syndrome, SAPS /I Simplified Acute Physiology Score II, VALRTI ventilator-associated respiratory tract infection; VAT, ventilatorassociated tracheobronchitis, VAP ventilator-associated pneumonia

a $p$ value for global comparison between the three groups (Gray's test considering extubations as competing events)

b $p$ values $<0.05$ for comparison with SARS-CoV-2 after applying Bonferroni correction

c Treating VAP as a competing event (in addition to extubations)

$d$ Treating VAT as a competing event (in addition to extubations)

e Adjusted for predefined confounders (age, gender, SAPS II, MacCabe classification, Immunosuppression, recent hospitalization, recent antibiotic, and ARDS), and calculated after handling missing values on covariates by multiple imputation $(m=20)$

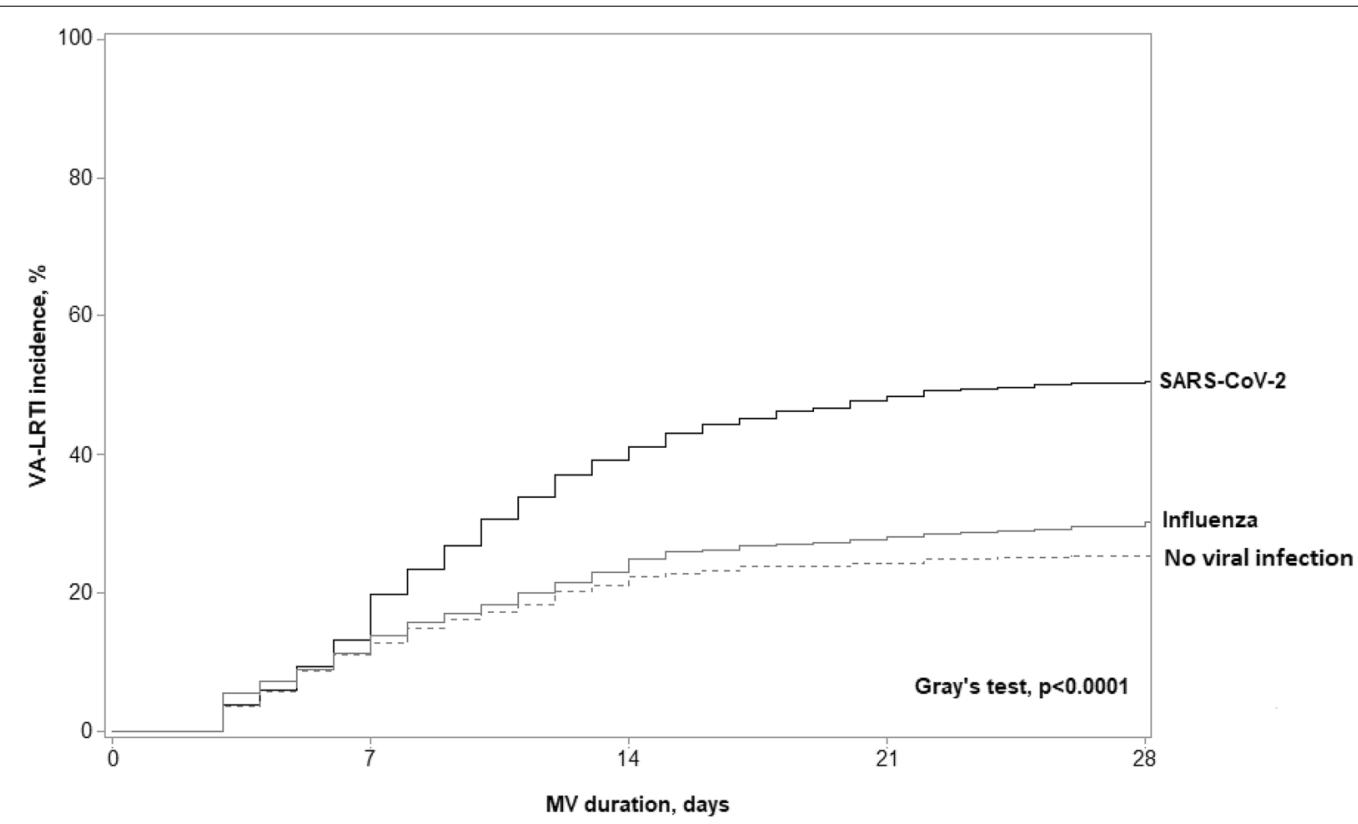

Fig. 1 The 28-day cumulative incidence of ventilator-associated lower respiratory tract infections. Cumulative incidence estimated using Kalbfleish and Prentice method, considering extubation (dead or alive) within 28 days as competing event. VA-LRT/ ventilator-associated respiratory tract infection, MV mechanical ventilation

ventilation, and higher incidence of ARDS, than in the two other groups. These factors were identified as independent risk factors for VAP. On the other hand, SARSCoV-2 pneumonia patients had lower severity scores at ICU admission, and less comorbidities than the two other groups. However, after careful adjustment for confounding factors, the incidence of VA-LRTI remained significantly higher in patients with SARS-CoV-2 pneumonia, as compared to the other groups. Nevertheless, as suggested by the less strong association between SARS-CoV-2 pneumonia and VA-LRTI using cause specific regression model, the higher incidence of VA-LRTI in patients with SARS-CoV-2 pneumonia is probably related, at least in part, to the longer duration of mechanical ventilation. Some immunosuppressive treatments commonly prescribed to patients with 
Table 4 Patient characteristics at the day of VALRTI diagnosis

\begin{tabular}{|c|c|c|c|c|c|c|}
\hline & \multicolumn{2}{|c|}{ SARS-CoV-2 pneumonia } & \multicolumn{2}{|c|}{ Influenza pneumonia } & \multicolumn{2}{|c|}{ No viral infection } \\
\hline & $\operatorname{VAT}(n=82)$ & $\operatorname{VAP}(n=205)$ & VAT $(n=39)$ & $\operatorname{VAP}(n=107)$ & VAT $(n=46)$ & $\operatorname{VAP}(n=87)$ \\
\hline SOFA score ${ }^{a}$ & $6(4-11)$ & $8(5-11)$ & $6(4-10)$ & $8(5-12)$ & $6(4-8)$ & $7(5-9)$ \\
\hline \multicolumn{7}{|l|}{ Diagnostic procedure } \\
\hline Endotracheal aspirates & $68 / 82(82.9)$ & $125 / 205$ (61.6) & 28/39 (71.8) & $59 / 105(56.2)$ & $35 / 46(77.8)$ & $62 / 87(72.9)$ \\
\hline Bronchoalveolar lavage & $14 / 82(17.1)$ & $78 / 205(38.4)$ & $11 / 39(28.2)$ & $46 / 105(43.8)$ & $10 / 46(22.2)$ & $23 / 87(27.1)$ \\
\hline Modified CPIS ${ }^{b}$ & $5(3-7)$ & $6(5-7)$ & $5(3-7)$ & $6(5-7)$ & $5(4-6)$ & $6(5-8)$ \\
\hline $\mathrm{PaO}_{2} / \mathrm{FiO}_{2}{ }^{\mathrm{C}}$ & $175(131-220)$ & $135(92-180)$ & $180(138-254)$ & $150(115-191)$ & $218(160-303)$ & $180(140-228)$ \\
\hline Antibiotic treatment & $67 / 82(81.7)$ & 191/205 (93.2) & $31 / 39(79.5)$ & 98/105 (93.3) & $36 / 46(78.3)$ & $82 / 87(94.3)$ \\
\hline Appropriate antibiotic treatment & $40 / 81(49.4)$ & $145 / 200(72.5)$ & $16 / 37(43.2)$ & $69 / 102(67.6)$ & 26/44 (59.1) & $54 / 87(62.1)$ \\
\hline
\end{tabular}

Data are presented as no./No.(\%) or median (interquartile range)

CPIS Clinical Pulmonary infection Score, $\mathrm{PaO}_{2} / \mathrm{FiO}_{2}$ arterial oxygen partial pressure/fractional inspired oxygen, SOFA Sequential Organ Failure Assessment

a 11 missing values

b 19 missing values

c 30 missing values

Table 5 Microorganisms responsible for ventilator-associated lower respiratory tract infections

\begin{tabular}{|c|c|c|c|}
\hline & $\begin{array}{l}\text { SARS-CoV-2 pneumonia } \\
(n=287)\end{array}$ & $\begin{array}{l}\text { Influenza pneumonia } \\
(n=146)\end{array}$ & $\begin{array}{l}\text { No viral infection } \\
(n=133)\end{array}$ \\
\hline Gram-positive cocci & $56(19.5)$ & $16(11)$ & $23(17.3)$ \\
\hline MSSA & $27(9.4)$ & $7(4.8)$ & $13(9.8)$ \\
\hline MRSA & $8(2.8)$ & $5(3.4)$ & $5(3.8)$ \\
\hline Enterococcus spp. & $9(3.1)$ & $2(1.4)$ & $2(1.5)$ \\
\hline Streptococcus pneumoniae & $8(2.8)$ & $1(0.7)$ & $2(1.5)$ \\
\hline Streptococcus spp. & $4(1.4)$ & $1(0.7)$ & $1(0.8)$ \\
\hline Gram-negative bacilli & $240(83.6)$ & $131(89.7)$ & $109(82)$ \\
\hline Pseudomonas aeruginosa & $64(22.3)$ & $33(23.1)$ & $23(17.3)$ \\
\hline Enterobacter spp. & $54(18.8)$ & $23(15.8)$ & $17(12.8)$ \\
\hline Klebsiella spp. & $33(11.5)$ & $21(14.4)$ & $21(15.8)$ \\
\hline Escherichia coli & $24(8.4)$ & $12(8.2)$ & $8(6.1)$ \\
\hline Acinetobacter baumannii & $21(7.3)$ & $22(15.1)$ & $14(10.5)$ \\
\hline Stenotrophomonas maltophilia & $10(3.5)$ & $3(2.1)$ & $7(5.3)$ \\
\hline Serratia marcescens & $9(3.1)$ & $2(1.4)$ & $6(4.5)$ \\
\hline Citrobacter freundii & $6(2.1)$ & $1(0.7)$ & $1(0.8)$ \\
\hline Citrobacter spp & $8(2.8)$ & $3(2.1)$ & $4(3)$ \\
\hline Proteus mirabilis & $5(1.7)$ & $1(0.7)$ & $1(0.8)$ \\
\hline Haemophilus influenza & $3(1)$ & $6(4.1)$ & $6(4.5)$ \\
\hline Morganella morganii & $3(1)$ & $4(2.7)$ & $1(0.8)$ \\
\hline Other & $15(5.2)$ & $9(6.2)$ & $5(3.8)$ \\
\hline Polymicrobial & $28(9.8)$ & $8(5.5)$ & $10(7.5)$ \\
\hline Multidrug-resistant isolates & $67(23.3)$ & $56(38.4)$ & $45(33.8)$ \\
\hline
\end{tabular}

Data are presented as $N(\%)$

MRSA methicillin-resistant Staphylococcus aureus, MSSA methicillin-sensitive Staphylococcus aureus

SARS-CoV-2 pneumonia, such as corticosteroids, might have also increased the risk for VA-LRTI. Although the rate of patients who received corticosteroids was not different between SARS-CoV-2 and influenza patients, the duration and dose of corticosteroids were higher in
SARS-CoV-2 patients than in the two other groups. Second, one would expect lower rates of ICU-acquired infection in patients with SARS-CoV-2 pneumonia because strict hygiene measures are used to avoid cross-transmission of the virus [14]. In contrast, previous studies 
reported that contact isolation measures, especially inappropriate glove use, could increase the transmission of MDR bacteria [23-25]. However, the lower rate of MDR bacteria among patients with VA-LRTI in SARS-CoV-2 pneumonia, as compared to the two other groups (23.3\%, $38.4 \%$, and $33.8 \%$; respectively) is against this hypothesis. Third, the surge of critically ill patients during the pandemic might have influenced the quality of critical care provided, and might have increased the risk for VA-LRTI. However, in some ICUs the ratio of nurse/patient was substantially increased and hygiene isolation measures were reinforced to protect other patients and healthcare workers. Another potential explanation would be specific pulmonary lesions related to SARS-CoV-2 infection. Whether ARDS related to COVID-19 is clinically different from ARDS related to other causes is a matter for debate [26]. However, histologic studies reported acute fibrinous and organizing pneumonia, characterized by an extensive intra-alveolar fibrin deposition and endothelial injury [27]. Distinctive vascular features, consisting of severe endothelial injury associated with the presence of intracellular virus and disrupted cell membranes was recently reported [28]. Widespread thrombosis distinguished the pulmonary pathophysiology of Covid-19, from that of equally severe influenza virus infection [28]. These lesions might promote local immunity alteration, bacterial colonization, and further lung infection [29]. However, further studies are required to confirm this hypothesis.

Although microorganisms responsible for VA-LRTI were comparable in the three groups, MDR bacteria incidence was lower in SARS-CoV-2 patients, as compared to the two other groups. This could be explained by the lower rate of recent antibiotic treatment and recent hospitalization in SARS-CoV-2 patients, as compared to the two other groups. However, duration of exposure to antimicrobials in the ICU was comparable in the three groups. Another possible explanation for the low incidence of MDR bacteria in SARS-CoV-2 patients is the better quality of hand hygiene in these patients [14].

To the best of our knowledge, this is the first study that evaluates the relationship between SARS-CoV-2 infection and the incidence of VA-LRTI in the critically ill patient. Strengths of our study are the large number of included patients, multicenter design, and the strict definition of VA-LRTI, requiring quantitative microbiological confirmation in all patients. However, some limitations of our study should be acknowledged. First, the study was retrospective and no blind external adjudication was performed to confirm VA-LRTI. However, all VA-LRTI were prospectively identified in all centers. Further, the presence of new infiltrate on chest X-ray was evaluated by at least two physicians. Second, we did not collect data on specific preventive measures in study patients, sedation and neuromuscular-agent use, or on compliance with hand-hygiene, and contact isolation measures. Third, all centers are located in Western Europe, mainly in France, with different distribution of MDR bacteria among participating countries. Therefore our results could not be generalized to other world regions. Fourth, ICU triage criteria, ICU efficiency, and isolation measures differed between the pandemic and the non-pandemic periods. However, microbiology criteria, sampling methods and VA-LRTI definition did not differ during the study period and were the same in all study groups. Further, the percentage of BAL/tracheal aspirate performed to diagnose VAT or VAP was quite similar between the three groups. During the pandemic it was impossible to have patients with diseases other than COVID-19, because ICUs were full with COVID-19 patients. Inclusions of patients with influenza or no viral infection, admitted to the ICU before the pandemic, allow a fair comparison of VALRTI incidence between COVID-19 patients and other at-risk patients. One could argue that the higher incidence of VA-LRTI in SARS-CoV-2 group, as compared to the other groups, is related to the higher number of microbiological sampling in these patients. Although the number of patients with suspected VA-LRTI was not collected, the higher mortality rate in patients with VAP as compared to those with no infection (data not shown) is against this hypothesis.

To conclude, the incidence of VA-LRTI is significantly higher in patients with SARS-CoV-2 pneumonia, as compared with patients with influenza pneumonia, or no viral infection at ICU admission. However, duration of mechanical ventilation was also higher in patients with SARS-CoV-2 pneumonia, as compared to other groups, and the association between the higher incidence of VA-LRTI and SARS-CoV-2 pneumonia may have not accounted for all relevant covariates. Efforts should be made to better understand the pathophysiology of VALRTI in patients with SARS-CoV-2 infection, and to improve preventive measures in this population.

\section{Electronic supplementary material \\ The online version of this article (https://doi.org/10.1007/s00134-020-06323-9) contains supplementary material, which is available to authorized users.}

\footnotetext{
Author details

${ }^{1}$ CHU de Lille, Médecine Intensive-Réanimation, 59000 Lille, France. ${ }^{2}$ Université de Lille, INSERM U995, Team Fungal Associated Invasive and Inflammatory Diseases, Lille Inflammation Research International Center, Lille, France. ${ }^{3}$ Department of Intensive Care Medicine, Multidisciplinary Intensive Care Research Organization (MICRO), St. James's Hospital, St. James Street, Dublin 8, Dublin, Eire, Ireland. ${ }^{4}$ Hospital Clinic, IDIBAPS, Universided de Barcelona, CIBERes, Barcelona, Spain. ${ }^{5}$ Polyvalent Intensive Care Unit, São Francisco Xavier Hospital, Centro Hospitalar de Lisboa Ocidental, and NOVA Medical School, CHRC, New University of Lisbon, Lisbon, Portugal. ${ }^{6}$ Center for Clinical Epidemiology and Research Unit of Clinical Epidemiology, OUH Odense
} 
University Hospital, Odense, Denmark. ${ }^{7}$ Intensive Care Unit, University Hospital of Larissa, University of Thessaly, Biopolis Larissa, 41110 Vólos, Greece. ${ }^{8}$ Critical Care Center, Corporacion Sanitaria Universitaria Parc Tauli, CIBER Enfermedades Respiratorias, Autonomous University of Barcelona, Parc Tauli 1, 08028 Sabadell, Spain. ${ }^{9}$ Service de Réanimation Polyvalente, Centre Hospitalier de Valenciennes, Valenciennes, France. ${ }^{10}$ Medical ICU, Amiens University Hospital, Amiens, France. ${ }^{11}$ Department of Medical Intensive Care, Caen University Hospital, 14000 Caen, France. ${ }^{12}$ Service de Réanimation et de Soins Intensifs, Centre Hospitalier de Douai, Route de Cambrai, Douai, France. ${ }^{13}$ Department of Intensive Care, François Mitterrand University Hospital, Dijon, France. ${ }^{14}$ Medical Intensive Care Unit, Rouen University Hospital, Normandie Université, UNIROUEN, Inserm U1096, FHU-REMOD-VHF, 76000 Rouen, France. ${ }^{15}$ ICU, Roubaix Hospital, Roubaix, France. ${ }^{16}$ Service de Médecine Intensive Réanimation, Institut de Cardiologie, Hôpital Pitié-Salpêtrière, Assistance Publique-Hôpitaux de Paris (APHP), Sorbonne Université, 47-83, Boulevard de I'Hôpital, 75651 Paris Cedex 13, France. ${ }^{17}$ Univ. Lille, CHU Lille, ULR 2694-METRICS: Évaluation des Technologies de santé et des Pratiques Médicales, 59000 Lille, France. ${ }^{18}$ Médecine Intensive Réanimation, Hôpital Saint Philibert GHICL, Université Catholique, Lille, France. ${ }^{19} \mathrm{CHU}$ de Poitiers, Médecine Intensive Réanimation, CIC 1402 ALIVE, Université de Poitiers, Poitiers, France. ${ }^{20}$ Service de Médecine Intensive et Réanimation, Hôpital Saint-Louis, 1 Avenue Claude Vellefaux, 75010 Paris, France. ${ }^{21}$ Département de Médecine Intensive-Réanimation, CHU d'Angers, Université d'Angers, 4 rue Larrey, 49933 Angers Cedex 9, France. ${ }^{22}$ Service de Médecine Intensive Réanimation, AP-HP, Hôpital Saint-Antoine, Assistance Publique-Hôpitaux de Paris, 184 rue du Faubourg Saint-Antoine, Paris, 75571 Cedex 12, France. ${ }^{23}$ Service de Pneumologie, Médecine Intensive, Réanimation (Département "R3S"), AP-HP, Sorbonne Université, Groupe Hospitalier Universitaire Pitié-Salpêtrière Charles Foix, 75013 Paris, France. ${ }^{24}$ Service de Médecine Intensive Réanimation, CHU de Tours, Hôpital Bretonneau, 2 Bd Tonnellé, 37000 Tours, France. ${ }^{25}$ 1st Department of Pulmonary Medicine and Intensive Care Unit, "Sotiria" Chest Hospital, National and Kapodistrian University of Athens, Athens, Greece. ${ }^{26}$ Service de Médecine Intensive, Réanimation, Hospices Civils de Lyon, Hôpital Edouard Herriot, 5, place d'Arsonval, 69437 Lyon Cedex 03, France. ${ }^{27}$ Medical Intensive Care Unit, Cochin Hospital, AP-HP, Centre, Université de Paris, Paris, France.

${ }^{28}$ 1st Department of Intensive Care Medicine, Evaggelismos Hospital, National and Kapodistrian University of Athens Medical School, Athens, Greece.

${ }^{29}$ Réanimation Médicale et Toxicologique, Hôpital Lariboisière, Université de Paris, INSERM UMRS-1 144, Paris, France. ${ }^{30}$ Intensive Care Unit, PellegrinTripode Hospital, University Hospital of Bordeaux, Bordeaux, France. ${ }^{31}$ Critical Care Department, Hospital Universitari Parc Taulí, Sabadell, Spain. ${ }^{32}$ Assistance Publique-Hôpitaux de Paris, CHU Henri Mondor, Service de réanimation médicale, Créteil, France. ${ }^{33}$ Service de Médecine Intensive Réanimation, CHU de Nantes, Nantes, France. ${ }^{34}$ Sorbonne Université, Assistance PubliqueHôpitaux de Paris, Service de Médecine Intensive Réanimation, Hôpital Tenon, Paris, France. ${ }^{35}$ Réanimation polyvalente, CH Lens, Lens, France. ${ }^{36}$ Réanimation Polyvalente, CH Victor Dupouy, Argenteuil, France. ${ }^{37}$ Intensive Care Unit, Hôpital de Béthune, 62408 Béthune, France. ${ }^{38}$ Service de Réanimation, Hôpital Duchenne, Rue Monod, 62200 Boulogne-sur-Mer, France. ${ }^{39}$ Sorbonne Université, AP-HP, Hôpital de la Pitié-Salpêtrière, Département de Neurologie, Unité de Médecine Intensive Réanimation Neurologique, Paris, France. ${ }^{40}$ Intensive Care Unit, Hospital Universitari Sagrat Cor, and Ciber de Enfermedades Respiratorias (Ciberes, CB06/06/0028)-Institut d'Investigacions Biomèdiques August Pi i Sunyer (IDIBAPS), Barcelona, Spain. ${ }^{41}$ Department of Pulmonology, Hospital Clinic Barcelona, University of Barcelona, IDIBAPS, CIBERES, ICREA, Barcelona, Spain.

\section{Acknowledgements}

Raphaël Favory: CHU de Lille, Centre de Réanimation, F-59000 Lille, France, Sébastien Preau: CHU de Lille, Centre de Réanimation, F-59000 Lille, France, Mercé Jourdain: CHU de Lille, Centre de Réanimation, F-59000 Lille, France, Julien Poissy: CHU de Lille, Centre de Réanimation, F-59000 Lille, France, Chaouki Bouras: Service de Réanimation polyvalente, Centre Hospitalier de Valenciennes, Valenciennes, France, Piehr Saint Leger: Service de Réanimation polyvalente, Centre Hospitalier de Valenciennes, Valenciennes, France, Hanane Fodil: Service de Réanimation polyvalente, Centre Hospitalier de Valenciennes, Valenciennes, France, François Aptel: Department of Intensive Care, François Mitterrand University Hospital, Dijon, France, Thierry Van Der Linden: Médecine intensive réanimation, Hôpital Saint Philibert GHICL, Université catholique, Lille, France, Arnaud W. Thille: CHU de Poitiers, Médecine Intensive Réanimation, CIC 1402 ALIVE, Université de Poitiers, Poitiers, France, Elie
Azoulay: Service de médecine intensive et réanimation, hôpital Saint-Louis, 1 Avenue Claude Vellefaux, 75010, Paris, France, Frédéric Pene: Medical Intensive Care Unit, Cochin Hospital, AP-HP. Centre, \& Université de Paris, Paris, France, Keyvan Razazi: Assistance Publique-Hôpitaux de Paris, CHU Henri Mondor, Service de réanimation médicale, Créteil, France, François Bagate: Assistance Publique-Hôpitaux de Paris, CHU Henri Mondor, Service de réanimation médicale, Créteil, France, Damien Contou: Réanimation Polyvalente, CH Victor Dupouy, Argenteuil, France, Guillaume Voiriot: Sorbonne Université, Assistance Publique-Hôpitaux de Paris, Service de Médecine Intensive Réanimation, Hôpital Tenon, Paris, France, Didier Thevenin: Réanimation polyvalente CH Lens, France, Bertrand Guidet: Service de Médecine Intensive Réanimation, AP-HP, Hôpital Saint-Antoine, Assistance Publique-Hôpitaux de Paris, 184 rue du Faubourg Saint-Antoine, Paris, 75571 Cedex 12, France, Loïc Le Guennec: Sorbonne Université, AP-HP, Hôpital de la Pitié-Sal pêtrière, Département de Neurologie, Unité de Médecine Intensive Réanimation Neurologique, Paris, France, Achille Kouatchet: Département de Médecine Intensive-Réanimation, CHU d'Angers, Université d'Angers, 4 rue Larrey, 49933, Angers Cedex 9, France, Stephan Ehrmann: Service de Médecine Intensive Réanimation, CHU de Tours, Hôpital Bretonneau, 2 Bd Tonnellé, 37000 Tours, France, Guillaume Brunin: Service de réanimation, hôpital Duchenne, rue Monod, 62200 Boulogne-sur-Mer, France, Elise Morawiec: Service de Pneumologie, Médecine intensive-Réanimation (Département "R3S"), AP-HP, Sorbonne Université, Groupe Hospitalier Universitaire Pitié-Sal pêtrière Charles Foix, 75013, Paris, France, Alexandre Boyer: Intensive Care Unit, Pellegrin-Tripode Hospital, University Hospital of Bordeaux, Bordeaux, France, Laurent Argaud: Service de Médecine Intensive-Réanimation, Hospices Civils de Lyon, Hôpital Edouard Herriot, 5, place d'Arsonval, 69437, Lyon Cedex 03, France, Sebastian Voicu: Réanimation Médicale et Toxicologique, Hôpital Lariboisière, Université de Paris, INSERM UMRS-1144, Paris, France, Ania Nieszkowska: Service de Médecine Intensive Réanimation, Institut de Cardiologie, Hôpital Pitié-Salpêtrière, Assistance Publique-Hôpitaux de Paris (APHP), Sorbonne Université, 47-83, Boulevard de l'Hôpital, 75651, Paris Cedex 13, France, Benjamin Kowalski: Service de réanimation et de soins intensifs, centre hospitalier de Douai, route de Cambrai, Douai, France, Gemma Goma: Critical Care Department, Hospital Universitari Parc Taulí Sabadell, Spain, Emilio Diaz: Critical Care Department, Hospital Universitari Parc Taulí, Sabadell, Spain, Luis Morales: Intensive Care Unit, Hospital Universitari Sagrat Cor, and Universidad Autonoma de Barcelona, Barcelona; Spain Intensive Care Unit, Hospital Universitari Sagrat Cor, Barcelona, Spain; Institut d'Investigacio i Innovacio Parc Tauli, Sabadell, Spain, Vassiliki Tsolaki: Critical Care Department, Larissa CHU, University of Thessaly, Biopolis Larissa, 41110 Greece, George Gtavriilidis: 1st Department of Pulmonary Medicine and Intensive Care Unit, National and Kapodistrian University of Athens, "Sotiria" Chest Hospital, Athens, Greece, Spyros D. Mentzelopoulos: 1st Department of Intensive Care Medicine, National and Kapodistrian University of Athens Medical School, Evaggelismos Hospital, Athens, Greece, David Nora: Polyvalent Intensive Care Unit, São Francisco Xavier Hospital, Centro Hospitalar de Lisboa Ocidental, Lisbon, Portugal, Sean Boyd: 3Department of Intensive Care Medicine, Multidisciplinary Intensive Care Research Organization (MICRO), St. James's Hospital, St. James Street, Dublin 8, Dublin, Eire, Ireland, Luis Coelho: Polyvalent Intensive Care Unit, São Francisco Xavier Hospital, Centro Hospitalar de Lisboa Ocidental, Lisbon, Portugal, Julien Maizel: Medical ICU, Amiens university hospital, Amiens, France, Damien Du Cheyron: Department of Medical Intensive Care, Caen University Hospital, F-14000 Caen, France, Mehdi Imouloudene: Service de réanimation et de soins intensifs, centre hospitalier de Douai, route de Cambrai, Douai, France, JeanPierre Quenot: Department of Intensive Care, François Mitterrand University Hospital, Dijon, France, Arnaud Guilbert: 42Medical Intensive Care Unit, Rouen University Hospital, Rouen, France, Catia Cilloniz: Pulmonology Department, Hospital Clinic of Barcelona, Ciber de Enfermedades Respiratorias (Ciberes, CB06/06/0028)-Institut d'Investigacions Biomèdiques August Pi i Sunyer (IDIBAPS), Barcelona, Spain

\section{Author contributions}

$A R, I M L, P P, J L, A D$, and $S N$ designed the study. $J L$, and $A D$ performed the statistical analyses. All authors collected study data. The first draft was written by $\mathrm{AR}$, and SN. All authors participated in writing, and revising the manuscript.

\section{Funding}

This study was supported in part by a grant from the French government through the «Programme Investissement d'Avenir» (I-SITE ULNE) managed by the Agence Nationale de la Recherche (coVAPid project). The funders of the 
study had no role in the study design, data collection, analysis, or interpretation, writing of the report, or decision to submit for publication.

\section{Compliance with ethical standards}

\section{Conflicts of interest}

$A R$ received personal fees from MaatPharma, IML received personal fees from MSD, and Gilead. AA received personal fees from Lilly Foundation, and grants from Grifols and Fischer \& Paykel. CEL received personal fees from Bayer, Merck, Aerogen, Biomérieux, ThermoFischer Brahms, and Carmat. NW received personal fees from MedDay pharmaceuticals. SN received personal fees from MSD, Bio Rad, BioMérieux, Gilead, and Pfizer. All other authors declare no competing interests.

\section{Data sharing}

All data needed to evaluate the conclusions in this Article are present and tabulated in the main text or the appendix. This article is the result of an original retrospective cohort. For individual de-identified raw data that underlie the results reported in this article, please contact the corresponding author.

\section{Publisher's Note}

Springer Nature remains neutral with regard to jurisdictional claims in published maps and institutional affiliations.

Received: 13 August 2020 Accepted: 11 November 2020 Published online: 3 January 2021

\section{References}

1. Argenziano MG, Bruce SL, Slater CL et al (2020) Characterization and clinical course of 1000 patients with COVID-19 in New York: retrospective case series. medRxiv Prepr Serv Heal Sci. https://doi. org/10.1101/2020.04.20.20072116

2. Lewnard JA, Liu VX, Jackson ML et al (2020) Incidence, clinical outcomes, and transmission dynamics of severe coronavirus disease 2019 in California and Washington: prospective cohort study. BMJ. https://doi. org/10.1136/BMJ.M1923

3. Docherty $A B$, Harrison EM, Green CA et al (2020) Features of 20133 UK patients in hospital with covid-19 using the ISARIC WHO Clinical Characterisation Protocol: prospective observational cohort study. BMJ. https:// doi.org/10.1136/BMJ.M1985

4. Martin-Loeches I, Povoa P, Rodríguez A et al (2015) Incidence and prognosis of ventilator-associated tracheobronchitis (TAVeM): a multicentre, prospective, observational study. Lancet Respir Med 3:859-868. https:// doi.org/10.1016/S2213-2600(15)00326-4

5. Papazian L, Klompas M, Luyt C-E (2020) Ventilator-associated pneumonia in adults: a narrative review. Intensive Care Med 46:888-906. https://doi. org/10.1007/s00134-020-05980-0

6. Nair GB, Niederman MS (2014) Ventilator-associated pneumonia: present understanding and ongoing debates. Intensive Care Med 41:34-48. https ://doi.org/10.1007/s00134-014-3564-5

7. Forel J-M, Voillet F, Pulina D et al (2012) Ventilator-associated pneumonia and ICU mortality in severe ARDS patients ventilated according to a lungprotective strategy. Crit Care 16:R65. https://doi.org/10.1186/cc11312

8. Chastre J, Fagon J-Y (2002) Ventilator-associated pneumonia. Am J Respir Crit Care Med 165:867-903. https://doi.org/10.1164/ajrccm.165.7.21050 78

9. Wu C, Chen X, Cai Y et al (2020) Risk factors associated with acute respiratory distress syndrome and death in patients with coronavirus disease 2019 pneumonia in Wuhan, China. JAMA Intern Med. https://doi. org/10.1001/jamainternmed.2020.0994

10. Mahmudpour M, Roozbeh J, Keshavarz M et al (2020) COVID-19 cytokine storm: the anger of inflammation. Cytokine. https://doi.org/10.1016/J. CYTO.2020.155151
11. Jeannet R, Daix T, Formento R et al (2020) Severe COVID-19 is associated with deep and sustained multifaceted cellular immunosuppression. Intensive Care Med. https://doi.org/10.1007/s00134-020-06127-x

12. Sinha P, Matthay MA, Calfee CS (2020) Is a cytokine storm; relevant to COVID-19? JAMA Intern Med. https://doi.org/10.1001/jamaintern med.2020.3313

13. Yang X, Yu Y, Xu J et al (2020) Clinical course and outcomes of critically ill patients with SARS-CoV-2 pneumonia in Wuhan, China: a single-centered, retrospective, observational study. Lancet Respir Med. https://doi. org/10.1016/S2213-2600(20)30079-5

14. Ye L, Yang S, Liu C (2020) Infection prevention and control in nursing severe coronavirus disease (COVID-19) patients during the pandemic. Crit Care. https://doi.org/10.1186/S13054-020-03076-1

15. Gu S, Chen Y, Wu Z et al (2020) Alterations of the gut microbiota in patients with COVID-19 or H1N1 influenza. Clin Infect Dis. https://doi. org/10.1093/CID/CIAA709

16. Zuo T, Zhang F, Lui GCY et al (2020) Alterations in gut microbiota of patients with COVID-19 during time of hospitalization. Gastroenterology. https://doi.org/10.1053/j.gastro.2020.05.048

17. Paul M, Shani V, Muchtar E et al (2010) Systematic review and meta-analysis of the efficacy of appropriate empiric antibiotic therapy for sepsis. Antimicrob Agents Chemother 54:4851-4863. https://doi.org/10.1128/ AAC.00627-10

18. Magiorakos A-P, Srinivasan A, Carey RB et al (2012) Multidrug-resistant, extensively drug-resistant and pandrug-resistant bacteria: an international expert proposal for interim standard definitions for acquired resistance. Clin Microbiol Infect 18:268-281. https://doi.org/10.111 1/j.1469-0691.2011.03570.x

19. Pintilie M (2002) Dealing with competing risks: testing covariates and calculating sample size. Stat Med 21:3317-3324. https://doi.org/10.1002/ sim.1271

20. Prentice RL, Kalbfleisch JD, Peterson AV et al (1978) The analysis of failure times in the presence of competing risks. Biometrics 34:541-554

21. van Buuren S, Groothuis-Oudshoorn K (2011) mice: multivariate Imputation by Chained Equations in R. J Stat Softw 45:1-67. https://doi. org/10.18637/jss.v045.i03

22. Gladitz J (1989) Rubin, Donald B.: Multiple Imputation for Nonresponse in Surveys. John Wiley \&amp; Sons, Chichester - New York - Brisbane Toronto - Singapore 1987, xxx, 258 S., 6 Abb., £ 30.25, ISSN 0271-6232. Biometrical J 31:131-132. doi: https://doi.org/10.1002/bimj.4710310118

23. Yap FHY, Gomersall CD, Fung KSC et al (2004) Increase in methicillinresistant Staphylococcus aureus acquisition rate and change in pathogen pattern associated with an outbreak of severe acute respiratory syndrome. Clin Infect Dis 39:511-516. https://doi.org/10.1086/422641

24. Harris AD, Pineles $L$, Belton B et al (2013) Universal glove and gown use and acquisition of antibiotic-resistant bacteria in the ICU: a randomized trial. JAMA 310:1571-1580. https://doi.org/10.1001/jama.2013.277815

25. Poulakou G, Nseir S, Daikos GL (2020) Less contact isolation is more in the ICU: pro. Intensive Care Med. https://doi.org/10.1007/s00134-020-06173 $-5$

26. Grasselli G, Tonetti T, Protti A et al (2020) Pathophysiology of COVID-19-associated acute respiratory distress syndrome: a multicentre prospective observational study. Lancet Respir Med. https://doi.org/10.1016/S2213 $-2600(20) 30370-2$

27. Copin M-C, Parmentier E, Duburcq T et al (2020) Time to consider histologic pattern of lung injury to treat critically ill patients with COVID-19 infection. Intensive Care Med 46:1124-1126. https://doi.org/10.1007/ s00134-020-06057-8

28. Ackermann M, Verleden SE, Kuehnel M et al (2020) Pulmonary vascular endothelialitis, thrombosis, and angiogenesis in Covid-19. N Engl J Med 383:120-128. https://doi.org/10.1056/NEJMoa2015432

29. Crane MJ, Lee KM, FitzGerald ES, Jamieson AM (2018) Surviving deadly lung infections: innate host tolerance mechanisms in the pulmonary system. Front Immunol 9:1421. https://doi.org/10.3389/fimmu.2018.01421 